\title{
Prediction of Immune checkpoint inhibitors benefit from routinely measurable peripheral blood parameters
}

\author{
Ioannis A. Voutsadakis ${ }^{1,2}$ \\ ${ }^{1}$ Algoma District Cancer Program, Sault Area Hospital, Sault Ste. Marie, Ontario, Canada; ${ }^{2}$ Section of Internal Medicine, Division of Clinical \\ Sciences, Northern Ontario School of Medicine, Sudbury, Ontario, Canada \\ Correspondence to: Ioannis A. Voutsadakis, MD, PhD. Algoma District Cancer Program, Sault Area Hospital, 750 Great Northern Road, Sault Ste \\ Marie, ON P6B 0A8, Canada. Email: ivoutsadakis@yahoo.com; ivoutsadakis@nosm.ca.
}

\begin{abstract}
Immunotherapy of cancer has been the most remarkable advance in cancer therapy in the last several years with the successful introduction of monoclonal antibody drugs that block inhibitory immune receptors to invigorate immune attack against tumors. With the introduction of these drugs the parallel need of predictive markers of response has arisen. Beyond markers from the tumor and the tumor micro-environment, the peripheral blood supplies several biomarkers for response that have been reported individually or in combinations to provide valuable predictive information. These include the number of circulating cell subsets, various ratios of them and combinations of factors that also encompass biochemical measurements such as LDH. Peripheral blood biomarkers are usually obtained during the routine patient evaluations, methodology is well-calibrated for the routine practice and their acquisition does not require extra infrastructure or financial resources. This paper will review available data on predictive markers for immune checkpoint inhibitors (ICIs) from peripheral blood, including biomarkers that have been less extensively studied. In addition, it will discuss ways forward for the use of peripheral blood biomarkers in immunotherapy prognostication.
\end{abstract}

Keywords: Immune checkpoint inhibitors (ICIs); biomarkers response prediction; ipilimumab; nivolumab; pembrolizumab

Submitted Nov 26, 2019. Accepted for publication Feb 24, 2020.

doi: $10.21037 /$ cco.2020.03.03

View this article at: http://dx.doi.org/10.21037/cco.2020.03.03

\section{Introduction}

Immune blockade inhibitors (also called immune checkpoint inhibitors; ICIs) represent monoclonal antibody drugs that function through reactivation of immune system to elicit an anticancer response. As a class these drugs bind and inhibit the function of inhibitory immune receptors (1). Immune checkpoint molecules that are inhibited by ICIs in clinical use currently include Cytotoxic T lymphocyte Antigen 4 (CTLA-4) and Programmed Death 1 (PD-1) and its ligand PD-L1. Other immune inhibitory receptor blockers, such as the macrophage inhibitor Hu5F9-G4 blocking the eat-menot receptor CD47, are in advanced development and will probably become available in the clinic, as data mature (2). ICIs holding approval for clinical use include CTLA-4 blockers ipilimumab and tremelimumab, PD-1 receptor blockers nivolumab, pembrolizumab and cemiplimab and PD-L1 blockers atezolizumab, avelumab and durvalumab. At least one checkpoint inhibitor has been approved for use alone or in combination with another checkpoint inhibitor or other drugs in a wide variety of malignancies. Examples are lung adenocarcinomas, squamous carcinomas and small cell lung cancers, urothelial and renal carcinomas, Hodgkin's lymphoma, melanoma and squamous carcinomas of the skin, head and neck cancers, triple negative breast cancers, as well as hepatocellular carcinoma and other gastrointestinal cancers (3-13). Moreover, the PD-1 inhibitor pembrolizumab has obtained a site-agnostic indication for treatment of any tumor with microsatellite instability (MSI), representing the first anti-neoplastic drug with approval not based on 
tumor site (14). ICIs have shown remarkable activity in the treatment of malignancies notoriously resistant or refractory to chemotherapy such as metastatic melanoma and renal carcinomas. They also outperform chemotherapy in subsets of other cancers such as lung carcinomas and urothelial carcinomas, at later line treatment $(3,5,15)$.

Immune blockade inhibitors have been studied in clinical trials for many common and less common cancers. In some occasions the observed activity was low and no regulatory approval for these drugs was obtained. In more responsive cancers, when non-selected populations are studied, the common pattern of response observed is a variable and many times low overall response rate while a long duration of response is obtained in a significant minority of responders. Rigorous research is underway in order to identify biomarkers predictive of response to ICIs. Some of those biomarkers have already been successfully incorporated into clinical practice. The best example is the target ligand of the PD-L1/PD-1 pair, PD-L1 which has been shown to predict higher responses with some of the ICIs blocking the pair and for some but not all tumor types. Another example is the presence of MSI or defects in Mismatch Repair proteins (dMMR) which forms the basis for a siteagnostic indication of pembrolizumab, as mentioned above. A significant minority of colorectal, endometrial and gastric cancers as well as rare cases of carcinomas from other locations harbor defects in MMR. Other biomarkers include the total number of mutations in the tumor referred to as tumor mutation burden (TMB) and the quantity and quality of immune cell infiltrates in the tumor microenvironment. These latter are not yet used in the clinic due to cost and standardization considerations. Related to the tumor immune infiltrates, subsets of circulating cells that may supply the tumor with immune infiltrates, guided by local cytokines have been studied and proposed to predict response to ICIs individually or in various combinations. Other measured indices such as Red cell Distribution Width or enzymes measured in the peripheral blood such as LDH have also been studied as predictors of response to ICIs.

This paper will review peripheral blood cell numbers and other elements of peripheral blood as predictors of response to ICIs with a focus to parameters which may be of importance but have been less studied.

\section{Lymphocytes, neutrophils, platelets and combinations}

The most abundant components of leukocytes, neutrophils and lymphocytes as well as platelets have been extensively studied as prognostic factors in several common cancers (16-23). More recently their value as predictors of ICIs response has been proposed separately or in combinations.

In general, patients with higher neutrophil and platelet counts and lower lymphocyte counts tend to fare worse and derive lower benefits from ICIs. A study of 108 melanoma patients that received nivolumab or pembrolizumab examined the predictive value of neutrophils, platelets and lymphocytes for PFS (24). Authors divided patients in three groups for each measured hematologic parameter and found that the intermediate group had not statistically significant different PFS than the worse group (higher counts for neutrophils and platelets and lower counts for lymphocytes). However, the group with the lower neutrophil counts and lower platelets counts and the group with the higher lymphocyte counts had a statistically significant better PFS than the worst group in each hematologic parameter category (24).

Another study that examined melanoma patients who received ipilimumab reported that among responders $94 \%$ of patients had a baseline lymphocyte count above $1 \times 10^{9} / \mathrm{L}$ while among non-responders only $78 \%$ of patients had a baseline lymphocyte count above $1 \times 10^{9} / \mathrm{L}(25)$. A study that analyzed melanoma patients who participated in prospective trials showed that higher lymphocytes counts both at baseline and before the third cycle of ipilimumab but also in the control arms receiving alternative treatments were associated with better survival (26). These results suggest that the absolute lymphocyte count may not be predictive of ipilimumab response but prognostic in all melanoma patients independently of therapy received.

The most studied combination of markers is the ratio of neutrophils to lymphocytes (NLR) which has been already the subject of several studies and at least two meta-analyses $(27,28)$. The most recent meta-analysis found higher NLR ratios to be associated with a hazard ratio (HR) for benefit from ICIs of 2.61 for OS (95\% confidence interval: 1.77-3.86) and a HR of 1.74 (95\% confidence interval: 1.34-2.27) for PFS compared with lower NLR ratios (28). Studies included in the meta-analysis used different cutoffs for classifying patients to the high or low ratio groups that ranged between 3 and 5.5. The ratio seems to predict benefit from ICIs in all cut-offs, although a higher cut-off (5 and higher) was associated with a higher HR suggesting that higher cut-offs were able to better separate the groups that benefited or did not benefit from ICIs. Studies included in the meta-analysis concerned patients treated 
with ipilimumab or nivolumab. Similar HR for OS with the two drugs were observed in the high and low neutrophil to lymphocyte ratio groups. Moreover, similar HR were observed in the studies that concerned melanoma patients and NSCLC patients. A single study that referred to metastatic renal cell carcinoma patients showed a higher HR for the prediction of inferior OS in the high neutrophil to lymphocyte ratio group (HR for OS of 5.3 versus 2.5 in the studies of NSCLC and melanoma) (29). Another study in 42 patients with metastatic renal cell carcinoma that received ICIs (11 of them in combination with VEGF drugs), and was not included in the above meta-analysis, showed worse PFS and OS in patients with NLR above 3 (30). The evolution (change) of NLR between baseline and the fourth treatment with nivolumab rather than a static NLR in time was examined in a cohort of 59 advanced NSCLC patients (31). Patients with a change of NLR of 1 or more progressed earlier and had a shorter OS than patients in whom NLR changed less. Others have reported that NLR of 5 or above after 2 cycles of nivolumab in NSCLC patients was predictive of worse OS (32). Moreover, nonresponders had more often an increase of NLR after 2 cycles of therapy compared to nivolumab responders. The same phenomenon was described in another small series of nivolumab treated NSCLC patients where NLR increases during therapy was more often observed in progressive disease (33).

The ratio of platelets to lymphocytes (PLR) was examined in a few further studies as an ICIs predictive marker. In a small study of 52 metastatic NSCLC patients receiving nivolumab both PLR and NLR were predictive of OS. Patients with ratios above the median had worse survival (34). A study of 54 NSCLC who were treated with nivolumab or pembrolizumab examined NLR, PLR and the product of NLR times platelets (which authors named Systemic Immune-Inflammation Index, SII) at baseline and at 6 weeks after initiation of treatment as predictors of response (35). None of the ratios was predictive at baseline. At 6 weeks after treatment initiation, NLR of less than 5 and SII of less than 730 were predictive of a higher response (35). In contrast PLR with a cut-off of 169 was not predictive even at 6 weeks. Another small study of 58 metastatic RCC receiving nivolumab examined NLR, PLR and monocyte to lymphocyte ratio (MLR) as predictive markers of survival and found higher MLR to be an independent factor for worse PFS and higher MLR as well as higher NLR to be independent factors for worse OS (36).

Overall, most, but not all, studies that examined circulating neutrophils, platelets and lymphocytes as predictive factors for ICIs efficacy confirm the positive predictive value of higher lymphocytes counts and the adverse predictive influence of higher neutrophil and platelet counts. Ratios that include these cells may improve their prognostic strength. However, defining the optimal cut-offs would be critical for improvement of predictive performance.

\section{Lymphocyte and monocyte subsets and activation status}

Peripheral lymphocyte sub-sets have been examined as prognosticators of PD-1 blockade response. In a study of melanoma patients that received pembrolizumab, baseline peripheral blood CD8+/PD-1+ cells that expressed a level of Ki67 proliferation marker above $6.5 \%$ were associated with systemic inflammation and lower OS (37). Interestingly, higher baseline aggregate tumor burden as measured from the total number of metastatic tumors present in patients was also associated with worse OS. The ratio of post-treatment level of Ki67 (3 weeks after starting pembrolizumab) in peripheral blood CD8+/PD-1+ cells to baseline tumor burden tended to correlate with response to therapy and OS.

CD4 cell sub-sets are also relevant for response to ICIs. Both an increase of CD8+ and of CD4+ lymphocytes after 4 and 8 weeks of ipilimumab treatment in melanoma were associated with better responses than patients that showed decreases in those lymphocytes at the same time period (38). CD4+/Foxp3-/PD- ${ }^{\text {high }}$ cells accumulate in tumor sites and may have a suppressive immune effect (39). In addition, these cells tend to increase in peripheral blood of patients treated with CTLA-4 inhibitors. When a PD-1 inhibitor is added in the treatment, responding patients display a decrease of CD4+/Foxp3-/PD-1 ${ }^{\text {high }}$ cells in periphery while non-responders do not show such a decrease. Authors suggest that in combination treatments PD-1 blockers should be administered in excess of CTLA- 4 drugs in order to maintain their capability to suppress CD4+/Foxp3-/PD$1^{\text {high }}$ cells (39).

An additional signature for lack of response to ICIs may include a phenotype of senescence in peripheral $\mathrm{T}$ cells with higher expression of TIM3 inhibitory receptor and lower CD28 co-stimulatory molecule (40). The activatory receptor ICOS was found to be commonly increasing in $\mathrm{CD}^{+} \mathrm{T}$ cells of patients treated with ipilimumab but whether this increase influences response to treatment 
remains unknown (41).

The monocytic subset of myeloid-derived suppressor cells (moMDSCs) a sub-set of MDSCs with the phenotype CD14 $/$ HLA-DR ${ }^{\text {low/negative }}$ tends to be higher in melanoma patients than in healthy donors (42). moMDSCs were examined in patients with metastatic melanomas treated with ipilimumab $(43,44)$. Patients with lower CD14 $/$ HLA$\mathrm{DR}^{\text {low/negative }}$ cell numbers in their peripheral blood at baseline and at 6 weeks of ipilimumab treatment had a longer OS than patients with higher CD14 $/$ HLA-DR ${ }^{\text {low/negative }}$ cell numbers (43). Patients that obtained a clinical benefit from therapy tended to have a decrease in circulating moMDSCs compared with patients with progressive disease that showed no decrease. Patients with moMDSCs that were less than $13.05 \%$ of the total circulating CD4+ lymphoid cells at 6 weeks of treatment had a better OS than counterparts with moMDSCs above the cut-off (HR 2.89, 95\% confidence interval: 1.59-6.99). Similarly, patients with a higher effector memory CD8+ phenotype (CD8+/ CD45RA-/CCR7-) at 9 weeks of treatment had better OS than patients with lower circulating effector memory CD8 cells (44). A subset of CD8+ effector memory (CD8+/ CD45RA-/CCR7-) cells characterized by positivity to CD27 and CD28 were also associated with response of melanoma patients to ipilimumab (45). Patients with this subset of CD8+ cells representing more than $13 \%$ of the CD8+ compartment had better Response Rate (RR) and OS than counterparts with lower CD8+/CD45RA-/CCR7-/ CD27+/CD28+ cells. In contrast, higher levels of terminally differentiated cells with the phenotype CD8+/CD45RA+/ CCR7-/CD27-/CD28- (representing more than 23.8\% of the CD8 compartment) were associated with worse OS and RR outcomes (45). Another study, though, suggested that the baseline ratio of central memory (CD45-/CCR7+) $\mathrm{T}$ cells to effector (CCR7-) T cells was associated with PFS in NSCLC patients receiving nivolumab (46). Patients with a higher ratio had better PFS. Authors suggested that this may be due to the association of higher central memory $\mathrm{T}$ cell to effector $\mathrm{T}$ cell ratios with an inflammatory signature and higher expression of PD-L1 in tumor.

Sub-sets of immune cells could vary in their ability to serve as predictive biomarkers of different ICIs treatment efficacy. This may be expected given that the mechanisms of anti-tumor invigoration and the sub-sets of immune cells expanded following therapy is distinct between CTLA-4 blockers and inhibitors of the PD-1/PD-L1 pair (47). Thus, memory $\mathrm{T}$ cell subsets seem to be more important for prediction in CTLA-4 blockade while NK cell expression of CD69 and MIP-1 $\beta$ could play a key role in PD-1 inhibition (48).

Use of immune cells sub-sets as described in this section has the drawback that they are not routinely used in practice and their validation would have to include analytical steps in clinical laboratories. This is important to consider in measurements that concern surface expression of proteins in circulating cells as these could be significantly influenced by ex vivo factors such as time from venipuncture to sample processing (42). Moreover, the optimal subset for prediction of ICIs effectiveness is unclear from the available data and may depend on the specific drugs. For these reasons, it is unlikely that lymphocyte and monocyte subsets will become useful clinical markers, despite their interest from a pathophysiologic point of view.

\section{Other hematologic parameters}

Other hematologic parameters, beyond neutrophils, lymphocytes and platelets, have been studied as predictors of ICIs response in cancer patients less frequently. The red cell distribution width (RDW), a measure of variability of red cell size (anisocytosis), has been proposed as a predictive marker in a study of 47 patients treated with nivolumab for stage III or metastatic NSCLC (49). In multivariate analysis, patients with RDW of $16 \%$ or above had a worse OS than patients with lower RDW. Authors examined also RDW in combination with NLR and reported that patients that had both high RDW and high NLR of 5 or above had the worse OS followed by patients that had only one of the two parameters elevated, while the group with both RDW below $16 \%$ and NLR below 5 had the best OS (49).

Monocytes were examined as prognostic markers for outcomes in a study of 32 metastatic NSCLC patients receiving nivolumab or pembrolizumab (50). Neither baseline monocytes nor neutrophils, that were examined in parallel, were predictive for response rate. However, responding patients with a higher level of peripheral monocytes above $0.7 \times 10^{9} / \mathrm{L}$ had a shorter time to response. In addition, patients with lower neutrophils below $4.2 \times 10^{9} / \mathrm{L}$ after the first dose of therapy had a higher response rate (50). Monocytes were also used together with lymphocytes for prognostication in melanoma patients treated with pembrolizumab (51). Patients with a lymphocyte to monocyte ratio above 1.7 had a better PFS and OS than counterparts with lymphocyte to monocyte ratio below 1.7 (51). As discussed in a previous section, lymphocytes together with monocytes were also predictive 
of PFS and OS in RCC patients treated with nivolumab (36).

Eosinophilia was observed more often in melanoma patients that received ICIs than in patients that were treated with other medications (52). An increase in eosinophilic count by at least $0.1 \times 10^{9} / \mathrm{L}$ between the first and second infusion of ipilimumab was noticed to be associated with improved OS in metastatic melanoma patients (53). This phenomenon was also observed in another melanoma cohort treated with ipilimumab where responders had a significant increase in eosinophil counts as opposed to nonresponding patients that had a less prominent increase in eosinophil counts (54). A higher than $1.1 \%$ relative eosinophilic count was also predictive of favorable OS in patients with advanced melanoma receiving ipilimumab and nivolumab combination therapy (55). In this cohort absolute monocyte counts below $0.8 \times 10^{9} / \mathrm{L}$, relative basophil count above $0.6 \%$, lower LDH (below 246) and lower NLR (below 4.73) were also associated with better OS. Another study on melanoma patients treated with ipilimumab showed higher absolute eosinophil counts, above $0.05 \times 10^{9} / \mathrm{L}$, to predict improved OS (56).

The relative eosinophilic count was found to be predictive of PFS and OS in patients with advanced head and neck cancers treated with nivolumab (57). The cutoff used was $1.5 \%$ and the group of patients with values above this cut-off had better PFS and OS compared with counterparts with values below the cut-off. In the same study, the relative neutrophil count and the relative lymphocyte count were significant for survival outcomes in the univariate analysis but lost significance in multivariate analysis. LDH was not predictive of outcomes in these patients (57). In NSCLC patients treated with nivolumab, absolute eosinophil counts above $0.15 \times 10^{9} / \mathrm{L}$ together with absolute lymphocyte counts above $1 \times 10^{9} / \mathrm{L}$ and absolute neutrophil counts below $7.5 \times 10^{9} / \mathrm{L}$ were predictive of better PFS and OS (58). Patients with two or three of these factors faired significantly better than counterparts with only one factor. This may have been due to the association of favorable factors with expression of PD-L1 in tumor. All patients with PD-L1 expression above 50\% had two or three factors present (58).

\section{Incorporation of biochemical measurements}

$\mathrm{LDH}$ is an enzyme that has been examined as a predictor of response to ICIs. In a study of advanced NSCLC patients, high LDH in combination with high (above 3) derived NLR (dNLR, defined as the ratio of neutrophils to white cells minus neutrophils) were predictive of worse PFS and OS in patients receiving PD-1 or PD-L1 blockers but not in patients receiving chemotherapy (59). In another study of NSCLC patients treated with nivolumab, high LDH was associated with absence of response to therapy (60). In melanoma patients receiving nivolumab, $\mathrm{LDH}$ values above 454 were associated with worse PFS and OS in multivariate analysis (61). Higher NLR was also predictive of worse outcomes in this cohort. In another melanoma cohort treated with ipilimumab, patients with LDH 2.3 times or more above normal had worse OS than patients with LDH between 1.2 to 2.3 times above normal or below 1.2 times above normal (56). In metastatic clear cell renal cell carcinoma treated with nivolumab, elevated serum LDH levels were associated with worse PFS compared with patients with normal LDH levels (62).

A study that used an index comprised of albumin, LDH and NLR (initially proposed as stratifying predictor for inclusion in phase I studies at the Institut Gustave-Roussy, France) as predictor of response to pembrolizumab, nivolumab or atezolizumab in 76 metastatic NSCLC patients found the index to be a predictor for OS but not PFS (63). In contrast, another index comprised of albumin, LDH and number of metastatic sites (The Royal Marsden Hospital Index) was predictive of PFS but not OS. When parameters were evaluated separately to find the most influential predictor, albumin and NLR but not LDH or number of metastatic sites were associated with OS (63). An index combining LDH with age and sex of the patient, previous ipilimumab treatment and presence of liver metastases was studied in melanoma patients for prediction of response to PD-1 targeted therapy (64). The index was confirmed to be predictive for response to treatment.

An index named ALI (Advanced Lung cancer Inflammation index) calculated as the product of albumin times BMI (Body Mass Index) divided by the NLR was investigated as prognostic factor in a series of 201 advanced NSCLC patients treated with nivolumab (65). Patients with an ALI below 18 had a significantly worse PFS and early progression on treatment. The same study confirmed that higher NLR was also associated with worse PFS.

A retrospective multicenter analysis of 616 metastatic melanoma patients who received pembrolizumab identified four peripheral blood parameters as being associated with longer OS (66). These included a baseline LDH value below 2.5 times the upper limit of normal, a relative eosinophil count above $1.5 \%$, a relative lymphocyte count above $17.5 \%$ and the absence of metastases in other organs 
beyond soft tissue and lung. Patients with all four factors had OS at 1 year of $83.9 \%$, while the 1 -year OS of patients with none of these factors present was $14.7 \%$ (66).

\section{Discussion}

Abnormalities of peripheral blood cell counts such as anemia, neutrophilia or thrombocytosis have been recognized as prognostic in cancer. In some cancers such as RCC these factors have been incorporated in widely used prognostic systems $(67,68)$. Cells of peripheral blood and other markers that may be measured in the blood could reflect aspects of the tumor immune microenvironment. The immune system receives clues from the tumor and responds by mobilizing immune cells of the innate and adaptive arms. Lymphocytes, neutrophils monocytes and NK cells are all mobilized by cytokines produced directly by cancer cells or indirectly by other cells that respond to signals from the tumor microenvironment. Moreover, other cells such as platelets and red cells are affected by signals emanating from the tumor or produced by the systemic inflammatory reaction that the tumor creates and their changes in number or size variability reflect this environment. Elevation of platelets may be a marker of immunosuppressive tumor micro-environment. Cancer Associated Fibroblasts (CAFs) secrete IL-6 which recruits myeloid derived suppressive cells and in addition promotes thrombopoiesis (69). Red cell anisocytosis as measured by RDW is also a marker of chronic inflammation and immune exhaustion. In HIV+ patients RDW correlates with high PD-1 expression in peripheral CD4+ lymphocytes (70).

With the introduction of ICIs of CTLA-4 and the PD-L1/PD-1 pair as active cancer immunotherapy, measurement of the tumor inflammation state has become an important element in prediction of cancers that will respond to these treatments. Tumors have been divided to cold or non-inflamed and hot or inflamed depending on their infiltration with cells of the immune system (71). Inflamed tumors have a better prognosis and respond better to ICIs therapy, especially if they produce a high number of neo-antigens, while most non-inflamed tumors do not respond to ICIs $(72,73)$. Immune presentation machinery may shape the quality of immune infiltrates in a tumor (74). Immune presentation machinery is also crucial for presenting neo-antigens that could be recognized by incoming immune effectors in order to mount an immune anti-tumor attack (75). Thus, tumor cells and immune cells are engaged in a two-way inter-relationship that eventually shapes the effectiveness of the anti-tumor immunity.

It is plausible that alterations in numbers of circulating blood cells are predictive of response to ICIs because they are associated or even produced by alterations of cytokines that have influence on the overall activity of immune system and on the number and function of effector lymphocytes. The previously mentioned association of thrombocytosis with IL-6, a well-described immunosuppressive cytokine, is a relevant example. However, a role of platelets in protecting tumor cells in transit and facilitating their homing by interaction with endothelial cells has been described (76). Cytokines producing eosinophilia such as IL-5 and eotaxin-1 (CCL11) may have tumor suppressive effects, hence the association of eosinophilia with tumor responses to ICIs (77). Nevertheless, IL-4, another cytokine associated with eosinophil production has protumorigenic effects (78). Administration of IL-2, used as immunotherapy in melanoma patients, results in elevation of IL-5 levels and eosinophilia (79). However, eosinophils may have anti-tumoral effects in the tumor micro-environment by promoting NK cells and $\mathrm{T}$ cells recruitment and direct cytotoxicity by production of granzymes and other cytotoxic proteins (80). Thus, both direct and indirect correlations of peripheral blood cells in tumor immunity may relate to their being prognostic and predictive factors in cancer. It is worth noticing that most of the data available on ICIs effectiveness prediction through peripheral blood elements lack comparative data on patients that received other therapies and strictly speaking represent arguments for these biomarkers being prognostic of the respective disease outcomes rather than solely predictive of immunotherapy outcomes. This fact is also supported by the known prognostic value of the biomarkers as studied in settings independent of therapy and by limited data from studies that included patients treated with other therapies (18-23,25). However, being concomitant cancer prognostic factors does not subtract from the clinical utility of peripheral blood biomarkers as predictive of immunotherapy response.

Currently several studies suggest that indices that consist of several types of cells and additional elements such as LDH or albumin may be more valuable than individual peripheral blood elements. However, the optimal number of elements that provide additive predictive value is not clear from the available data. The optimal manner to combine data and specifically whether indices that calculate ratios as opposed to predictive tools that use addition of abnormal findings in their calculations of risk 
is also unclear. An additional element in the predictive associations from certain studies has been the timing of evaluation and specifically the value of baseline as opposed to later measurements or even the value of dynamic evaluations of changes between baseline and later time points in predicting outcomes. Of course, the preferable time point of a biomarker would be at baseline where the prediction information is obtained before use of the drug. Alternatively, a dynamic time point using, for example, in addition to the baseline measurements, biomarker measurements before the second drug administration could constitute a quite relevant clinical tool if prediction obtained by the addition of the later measurement is robust.

The ultimate point to consider in further advancing the field of immunotherapy prediction by peripheral blood biomarkers and indeed of any other biomarkers is whether it is preferable to study and develop predictors of specific drugs or inhibitors of specific checkpoint molecules or a more generic approach could offer useful prediction for all ICIs. Most of the peripheral blood biomarkers examined appear to predict outcomes in diverse type of cancers and their predictive value does not seem to depend on the specific tumor.

Measurement of cell numbers and other relevant data from peripheral blood appear to add information on inflammation and mobilization of immune effectors in a systemic level and could contribute to a better prognostication of ICIs possibly serving as an adjunct to other currently used biomarkers such as PD-L1 expression in tumors and presence of MSI. A combination of those established biomarkers with other markers from the tumor and its micro-environment, such as total mutation burden and tumor infiltrating lymphocytes, as well as biomarkers from the peripheral blood may eventually become the optimal means of ICIs prognostication.

\section{Acknowledgments}

Funding: None.

\section{Footnote}

Conflicts of Interest: The author has completed the ICMJE uniform disclosure form (available at http://dx.doi. org/10.21037/cco.2020.03.03). The author has no conflicts of interest to declare.

Ethical Statement: The author is accountable for all aspects of the work in ensuring that questions related to the accuracy or integrity of any part of the work are appropriately investigated and resolved.

Open Access Statement: This is an Open Access article distributed in accordance with the Creative Commons Attribution-NonCommercial-NoDerivs 4.0 International License (CC BY-NC-ND 4.0), which permits the noncommercial replication and distribution of the article with the strict proviso that no changes or edits are made and the original work is properly cited (including links to both the formal publication through the relevant DOI and the license). See: https://creativecommons.org/licenses/by-nc-nd/4.0/.

\section{References}

1. Voutsadakis IA. Immune blockade inhibition in breast cancer. Anticancer Res 2016;36:5607-22.

2. Advani R, Flinn I, Popplewell L, et al. CD47 blockade by Hu5F9-G4 and rituximab in Non-Hodgkin's lymphoma. New Engl J Med 2018;379:1711-21.

3. Borghaei H, Paz-Ares L, Horn L, et al. Nivolumab versus docetaxel in advanced nonsquamous Non-Small-Cell Lung Cancer. N Engl J Med 2015;373:1627-39.

4. Garon EB, Rizvi NA, Hui R, et al. Pembrolizumab for the treatment of Non-Small-Cell Lung Cancer. N Engl J Med 2015;372:2018-28.

5. Brahmer J, Reckamp KL, Baas P, et al. Nivolumab versus docetaxel in advanced squamous-cell Non-Small-Cell Lung Cancer. N Engl J Med 2015;373:123-35.

6. Robert C, Long GV, Brady B, et al. Nivolumab in previously untreated melanoma without BRAF mutation. N Engl J Med 2015;372:320-30.

7. Powles T, Eder JP, Fine GD, et al. MPDL3280A (antiPD-L1) treatment leads to clinical activity in metastatic bladder cancer. Nature 2014;515:558-62.

8. Motzer RJ, Escudier B, McDermott DF, et al. Nivolumab versus everolimus in advanced renal-cell carcinoma. $\mathrm{N}$ Engl J Med 2015;373:1803-13.

9. Chen R, Zinzani PL, Fanale MA, et al. Phase II study of the efficacy and safety of pembrolizumab for relapsed/ refractory classic Hodgkin lymphoma. J Clin Oncol 2017;35:2125-32.

10. Migden MR, Rischin D, Schmults CD, et al. PD-1 blockade with cemiplimab in advanced cutaneous squamous-cell carcinoma. N Engl J Med 2018;379:341-51.

11. Kudo T, Hamamoto Y, Kato K, et al. Nivolumab treatment for oesophageal squamous-cell carcinoma: 
an open-label, multicentre, phase 2 trial. Lancet Oncol 2017;18:631-9.

12. Schmid P, Adams S, Rugo HS, et al. Atezolizumab and nab-paclitaxel in advanced triple-negative breast cancer. $\mathrm{N}$ Engl J Med 2018;379:2108-21.

13. Shitara K, Özgüroğlu M, Bang Y-J, et al. Pembrolizumab versus paclitaxel for previously treated, advanced gastric or gastro-oesophageal junction cancer (KEYNOTE-061): a randomised, open-label, controlled, phase 3 trial. Lancet 2018;392:123-33.

14. Lemery S, Keegan P, Pazdur R. First FDA approval agnostic of cancer site- when a biomarker defines the indication. New Engl J Med 2017;377:1409-12.

15. Bellmunt J, de Wit R, Vaughn DJ, et al. Pembrolizumab as second-line therapy for advanced urothelial carcinoma. N Engl J Med 2017;376:1015-26.

16. Stravodimou A, Voutsadakis IA. Pre-treatment thrombocytosis as a prognostic marker in metastatic breast cancer. Int J Breast Cancer 2013;2013:289563.

17. Stone RL, Nick AM, McNeish IA, et al. Paraneoplastic thrombocytosis in ovarian cancer. New Engl J Med 2012;366:610-8.

18. Digklia A, Voutsadakis IA. Thrombocytosis as a prognostic marker in stage III and IV serous ovarian cancer. Obstet Gynecol Sci 2014;57:457-63.

19. Policicchio A, Mercier J, Digklia A, Voutsadakis IA. Platelet and neutrophil counts as predictive markers of neoadjuvant therapy efficacy in rectal cancer. J Gastrointest Cancer 2019;50:894-900.

20. Mercier J, Voutsadakis IA. The platelets-neutrophils to lymphocytes ratio: A new prognostic marker in metastatic colorectal cancer. J Gastrointest Oncol 2018;9:478-86.

21. Mercier J, Voutsadakis IA. Comparison of hematologic and other prognostic markers in metastatic colorectal cancer. J Gastrointest Cancer 2019;50:493-506.

22. Steele M, Voutsadakis IA. Pre-treatment platelet counts as a prognostic and predictive factor in stage II and III rectal adenocarcinoma. World J Gastrointest Oncol 2017;9:42-9.

23. Voutsadakis IA. Thrombocytosis as a prognostic marker in gastrointestinal cancers. World J Gastrointest Oncol 2014;6:34-40.

24. Pan M, Alavi M, Herrinton LJ. Association of Inflammatory Markers with Disease Progression in Patients with Metastatic Melanoma Treated with Immune Checkpoint Inhibitors. Perm J 2018;22:17-149.

25. Simeone E, Gentilcore G, Giannarelli D, et al. Immunological and biological changes during ipilimumab treatment and their potential correlation with clinical response and survival in patients with advanced melanoma. Cancer Immunol Immunother 2014;63:675-83.

26. Postow MA, Chasalow SD, Kuk D, et al. Absolute lymphocyte count as a prognostic biomarker for overall survival in patients with advanced melanoma treated with ipilimumab. Melanoma Res 2020;30:71-5.

27. Sacdalan DB, Lucero JA, Sacdalan DL. Prognostic utility of baseline neutrophil-to-lymphocyte ratio in patients receiving immune checkpoint inhibitors: a review and meta-analysis. Onco Targets Ther 2018;11:955-65.

28. Xie X, Liu J, Yang H, et al. Prognostic value of baseline Neutrophil-to-Lymphocyte Ratio in outcome of immune checkpoint inhibitors. Cancer Invest 2019;37:265-74.

29. Bilen MA, Almeida Dutcher GM, Liu Y, et al. Association Between Pretreatment Neutrophil-to Lymphocyte Ratio and Outcome of Patients with Metastatic Renal-Cell Carcinoma Treated with Nivolumab. Clin Genitourin Cancer 2018;16:e563-75.

30. Jeyakumar G, Kim S, Bumma N, et al. Neutrophil lymphocyte ratio and duration of prior anti-angiogenic therapy as biomarkers in metastatic RCC receiving immune checkpoint inhibitor therapy. J Immunother Cancer 2017;5:82.

31. Dusselier M, Deluche E, Delacourt N, et al. Neutrophilto-lymphocyte ratio evolution is an independent predictor of early progression of second-line nivolumab-treated patients with advanced non-small-cell lung cancers. PLoS ONE 2019;14:e0219060.

32. Khunger M, Patil PD, Khunger A, et al. Post-treatment changes in hematological parameters predict response to nivolumab monotherapy in non-small cell lung cancer patients. PLoS One 2018;13:e0197743.

33. Kiriu T, Yamamoto M, Nagano T, et al. The time-series behavior of neutrophil-to-lymphocyte ratio is useful as a predictive marker in non-small cell lung cancer. PLoS One 2018;13:e193018.

34. Diem S, Schmid S, Krapf M, et al. Neutrophil-toLymphocyte ratio (NLR) and Platelet-to-Lymphocyte ratio (PLR) as prognostic markers in patients with nonsmall cell lung cancer (NSCLC) treated with nivolumab. Lung Cancer 2017;111:176-81.

35. Suh KJ, Kim SH, Kim YJ, et al. Post-treatment neutrophil to lymphocyte ratio at week 6 is prognostic in patients with advanced non-small cell lung cancers treated with anti-PD1 antibody. Cancer Immunol Immunother 2018;67:459-70.

36. Ishihara $\mathrm{H}$, Tashibana $\mathrm{H}$, Takagi $\mathrm{T}$, et al. Predictive impact of peripheral blood markers and C-Reactive 
Protein in nivolumab therapy for metastatic renal cell carcinoma. Target Oncol 2019;14:453-63.

37. Huang AC, Postow MA, Orlowski RJ, et al. T-cell invigoration to tumour burden ratio associated with antiPD-1 response. Nature 2017;545:60-5.

38. Martens A, Wistuba-Hamprecht K, Yuan J, et al. Increases in Absolute Lymphocytes and Circulating CD4+ and CD8+ T Cells Are Associated with Positive Clinical Outcome of Melanoma Patients Treated with Ipilimumab. Clin Cancer Res 2016;22:4848-58.

39. Zappasodi R, Budhu S, Hellmann MD, et al. Nonconventional Inhibitory CD4+Foxp3-PD-1hi T Cells as a Biomarker of Immune Checkpoint Blockade Activity. Cancer Cell 2018;33:1017-32.e7.

40. Moreira A, Gross S, Kirchberger MC, et al. Senescence markers: Predictive for response to checkpoint inhibitors. Int J Cancer 2019;144:1147-50.

41. Ng Tang D, Shen Y, Sun J, et al. Increased Frequency of ICOS+ CD4 T Cells as a Pharmacodynamic Biomarker for Anti-CTLA-4 Therapy. Cancer Immunol Res 2013;1:229-34.

42. Meyer C, Cagnon L, Costa-Nunes CM, et al. Frequencies of circulating MDSC correlate with clinical outcome of melanoma patients treated with ipilimumab. Cancer Immunol Immunother 2014;63:247-57.

43. Kitano S, Postow MA, Ziegler CGK, et al. Computational Algorithm-Driven Evaluation of Monocytic MyeloidDerived Suppressor Cell Frequency for Prediction of Clinical Outcomes. Cancer Immunol Res 2014;2:812-21.

44. de Coaña YP, Wolodarski M, Poschke I, et al. Ipilimumab treatment decreases monocytic MDSCs and increases CD8 effector memory $T$ cells in long-term survivors with advanced melanoma. Oncotarget 2017;8:21539-53.

45. Wistuba-Hamprecht K, Martens A, Heubach F, et al. Peripheral CD8 effector-memory type $1 \mathrm{~T}$-cells correlate with outcome in ipilimumab-treated stage IV melanoma patients. Eur J Cancer 2017;73:61-70.

46. Manjarrez-Orduño N, Menard LC, Kansal S, et al. Circulating T Cell Subpopulations correlate with Immune Responses at the Tumor Site and Clinical Response to PD1 Inhibition in Non-Small Cell Lung Cancer. Front Immunol 2018;9:1613.

47. Wei SC, Levine JH, Cogdill AP, et al. Distinct cellular mechanisms underlie anti-CTLA-4 and anti-PD-1 checkpoint blockade. Cell 2017;170:1120-33.e17.

48. Subrahmanyam PB, Dong Z, Gusenleitner D, et al. Distinct predictive biomarker candidates for response to anti-CTLA-4 and anti-PD-1 immunotherapy in melanoma patients. J Immunother Cancer 2018;6:18.

49. Kiriu T, Yamamoto M, Nagano T, et al. Prognostic value of red blood cell distribution width in Non-small Cell Lung Cancer treated with anti-programmed cell Death-1 antibody. In Vivo 2019;33:213-20.

50. Parikh K, Kumar A, Ahmed J, et al. Peripheral monocytes and neutrophils predict response to immune checkpoint inhibitors in patients with metastatic non-small cell lung cancer. Cancer Immunol Immunother 2018;67:1365-70.

51. Failing JJ, Yan Y, Porrata LF, Markovic SN. Lymphocyteto-monocyte ratio is associated with survival in pembrolizumab-treated metastatic melanoma patient. Melanoma Res 2017;27:596-600.

52. Moreira A, Leisgang W, Schuler G, Heinzerling L. Eosinophilic count as a biomarker for prognosis of melanoma patients and its importance in the response to immunotherapy. Immunotherapy 2017;9:115-21.

53. Delyon J, Mateus C, Lefeuvre D, et al. Experience in daily practice with ipilimumab for the treatment of patients with metastatic melanoma: an early increase in lymphocyte and eosinophil counts is associated with improved survival. Ann Oncol 2013;24:1697-703.

54. Gebhardt C, Sevko A, Jiang H, et al. Myeloid Cells and Related Chronic Inflammatory Factors as Novel Predictive Markers in Melanoma Treatment with Ipilimumab. Clin Cancer Res 2015;21:5453-9.

55. Rosner S, Kwong E, Shoushtari AN, et al. Peripheral blood clinical laboratory variables associated with outcomes following combination nivolumab and ipilimumab immunotherapy in melanoma. Cancer Med 2018;7:690-7.

56. Martens A, Wistuba-Hamprecht K, Geukes Foppen M, et al. Baseline Peripheral Blood Biomarkers Associated with Clinical Outcome of Advanced Melanoma Patients Treated with Ipilimumab. Clin Cancer Res 2016;22:2908-18.

57. Nishikawa D, Suzuki H, Koide Y, et al. Prognostic Markers in Head and Neck Cancer Patients Treated with Nivolumab. Cancers 2018;10:466.

58. Tanizaki J, Haratani K, Hayashi H, et al. Peripheral Blood Biomarkers Associated with Clinical Outcome in NonSmall Cell Lung Cancer Patients Treated with Nivolumab. J Thorac Oncol 2018;13:97-105.

59. Mezquita L, Auclinn E, Ferrara R, et al. Association of the Lung Immune Prognostic Index with Immune Checkpoint Inhibitor Outcomes in Patients with Advanced Non-Small Cell Lung Cancer. JAMA Oncol 2018;4:351-7

60. Agulló-Ortuño MT, Gómez-Martín Ó, Ponce S, et al. Blood predictive biomarkers for patients with Non-small- 
cell Lung Cancer associated with clinical response to nivolumab. Clin Lung Cancer 2020;21:75-85.

61. Capone M, Giannarelli D, Mallardo D, et al. Baseline neutrophil-to-lymphocyte ratio (NLR) and derived NLR could predict overall survival in patients with advanced melanoma treated with nivolumab. J Immunother Cancer 2018;6:74.

62. Shirotake S, Takamatsu K, Mizuno R, et al. Serum Lactate Dehydrogenase before nivolumab treatment could be a therapeutic prognostic biomarker for patients with metastatic clear cell renal cell carcinoma. Anticancer Res 2019;39:4371-7.

63. Minami S, Ihara S, Ikuta S, Komuta K. Gustave Roussy Immune Score and Royal Marsden Hospital Prognostic Score Are Biomarkers of Immune-Checkpoint Inhibitor for Non-Small Cell Lung Cancer. World J Oncol 2019;10:90-100.

64. Nosrati A, Tsai KK, Goldinger SM, et al. Evaluation of clinicopathological factors in $\mathrm{PD}-1$ response: derivation and validation of a prediction scale for response to PD-1 monotherapy. Br J Cancer 2017;116:1141-7.

65. Shiroyama T, Suzuki H, Tamiya M, et al. Pretreatment advanced lung cancer inflammation index (ALI) for predicting early progression in nivolumab-treated patients with advanced non-small cell lung cancer. Cancer Med 2018;7:13-20.

66. Weide B, Martens A, Hassel JC, et al. Baseline Biomarkers for Outcome of Melanoma Patients Treated with Pembrolizumab. Clin Cancer Res 2016;22:5487-96.

67. Motzer RJ, Bacik J, Murphy BA, et al. Interferon-alfa as a comparative treatment for clinical trials of new therapies against advanced renal cell carcinoma. J Clin Oncol 2002;20:289-96.

68. Heng DY, Xie W, Regan MM, et al. Prognostic factors for overall survival in patients with metastatic renal cell carcinoma treated with vascular endothelial growth factortargeted agents: Results from a large, multicenter study. J Clin Oncol 2009;27:5794-9.

69. Liu H, Shen J, Lu K. IL-6 and PD-L1 blockade combination inhibits hepatocellular carcinoma cancer

Cite this article as: Voutsadakis IA. Prediction of Immune checkpoint inhibitors benefit from routinely measurable peripheral blood parameters. Chin Clin Oncol 2020;9(2):19. doi: $10.21037 /$ cco.2020.03.03 development in mouse model. Biochem Biophys Res Commun 2017;486:239-44.

70. Al-Kindi SG, Zidar DA, McComsey GA, et al. Association of anisocytosis with markers of immune activation and exhaustion in treated HIV. Pathog Immun 2017;2:138-50.

71. Maleki Vareki S. High and low mutational burden tumors versus immunologically hot and cold tumors and response to immune checkpoint inhibitors. J Immunother Cancer 2018;6:157.

72. Jiang W, Liu K, Guo Q, et al. Tumor-infiltrating immune cells and prognosis in gastric cancer: a systematic review and meta-analysis. Oncotarget 2017;8:62312-29.

73. Rosenthal R, Larose Cadieux E, Salgado R, et al. Neoantigen-directed immune escape in lung cancer evolution. Nature 2019;567:479-85.

74. Park HS, Cho U, Im SY, et al. Loss of human leukocyte antigen class I expression is associated with poor prognosis in patients with advanced breast cancer. J Pathol Transl Med 2019;53:75-85.

75. Voutsadakis IA. Polymerase epsilon mutations and concomitant $\beta 2$-microglobulin mutations in cancer. Gene 2018;647:31-8.

76. Buergy D, Wenz F, Groden C, Brockmann MA. Tumor-platelet interaction in solid tumors. Int J Cancer 2012;130:2747-60

77. Umansky V, Utikal J, Gebhardt C. Predictive immune markers in advanced melanoma patients treated with ipilimumab. Oncoimmunology 2016;5:e1158901.

78. Ito SE, Shirota H, Kasahara Y, et al. IL-4 blockade alters the tumor microenvironment and augments the response to cancer immunotherapy in a mouse model. Cancer Immunol Immunother 2017;66:1485-96.

79. van Haelst Pisani C, Kovach JS, Kita H, et al. Administration of interleukin-2 (IL-2) results in increased plasma concentrations of IL-5 and eosinophilia in patients with cancer. Blood 1991;78:1538-44.

80. Simon SCS, Utikal J, Umansky V. Opposing roles of eosinophils in cancer. Cancer Immunol Immunother 2019;68:823-33. 